\title{
A New Criterion for Clusters Validation
}

\author{
Hosein Alizadeh, Behrouz Minaei, and Hamid Parvin \\ School of Computer Engineering, Iran University of Science and Technology (IUST), \\ Tehran, Iran \\ \{halizadeh, b_minaei,parvin\}@iust.ac.ir
}

\begin{abstract}
In this paper a new criterion for clusters validation is proposed. This new cluster validation criterion is used to approximate the goodness of a cluster. The clusters which satisfy a threshold of this measure are selected to participate in clustering ensemble. For combining the chosen clusters, a co-association based consensus function is applied. Since the Evidence Accumulation Clustering method cannot derive the co-association matrix from a subset of clusters, a new EAC based method which is called Extended EAC, EEAC, is applied for constructing the co-association matrix from the subset of clusters. Employing this new cluster validation criterion, the obtained ensemble is evaluated on some well-known and standard data sets. The empirical studies show promising results for the ensemble obtained using the proposed criterion comparing with the ensemble obtained using the standard clusters validation criterion.
\end{abstract}

Keywords: Clustering Ensemble, Stability Measure, Cluster Evaluation.

\section{Introduction}

Data clustering or unsupervised learning is an important and very difficult problem. The objective of clustering is to partition a set of unlabeled objects into homogeneous groups or clusters [3]. Clustering techniques require the definition of a similarity measure between patterns. Since there is no prior knowledge about cluster shapes, choosing a specific clustering method is not easy [12]. Studies in the last few years have tended to combinational methods. Cluster ensemble methods attempt to find better and more robust clustering solutions by fusing information from several primary data partitionings [8].

Fern and Lin [4] have suggested a clustering ensemble approach which selects a subset of solutions to form a smaller but better-performing cluster ensemble than using all primary solutions. The ensemble selection method is designed based on quality and diversity, the two factors that have been shown to influence cluster ensemble performance. This method attempts to select a subset of primary partitions which simultaneously has both the highest quality and diversity. The Sum of Normalized Mutual Information, SNMI [5]-[7] and [13] is used to measure the quality of an individual partition with respect to other partitions. Also, the Normalized Mutual Information, NMI, is employed for measuring the diversity among partitions. Although the ensemble size in this method is relatively small, this method achieves 
significant performance improvement over full ensembles. Law et al. proposed a multi objective data clustering method based on the selection of individual clusters produced by several clustering algorithms through an optimization procedure [10]. This technique chooses the best set of objective functions for different parts of the feature space from the results of base clustering algorithms. Fred and Jain [7] have offered a new clustering ensemble method which learns the pairwise similarity between points in order to facilitate a proper partitioning of the data without the a priori knowledge of the number of clusters and of the shape of these clusters. This method which is based on cluster stability evaluates the primary clustering results instead of final clustering.

Alizadeh et al. have discussed the drawbacks of the common approaches and then have proposed a new asymmetric criterion to assess the association between a cluster and a partition which is called Alizadeh-Parvin-Moshki-Minaei criterion, APMM. The APMM criterion compensates the drawbacks of the common method. Also, a clustering ensemble method is proposed which is based on aggregating a subset of primary clusters. This method uses the Average APMM as fitness measure to select a number of clusters. The clusters which satisfy a predefined threshold of the mentioned measure are selected to participate in the clustering ensemble. To combine the chosen clusters, a co-association based consensus function is employed [1].

\section{Proposed Method}

The main idea of our proposed clustering ensemble framework is utilizing a subset of best performing primary clusters in the ensemble, rather than using all of clusters. Only the clusters which satisfy a stability criterion can participate in the combination. The cluster stability is defined according to Normalized Mutual Information, NMI.

The manner of computing stability is described in the following sections in detail. After, a subset of the most stable clusters is selected for combination. This is simply done by applying a stability-threshold to each cluster. In the next step, the selected clusters are used to construct the co-association matrix. Several methods have been proposed for combination of the primary results [2] and [13]. In our work, some clusters in the primary partitions may be absent (having been eliminated by the stability criterion). Since the original EAC method [5] cannot truly identify the pairwise similarity while there is only a subset of clusters, we present a new method for constructing the co-association matrix. We call this method: Extended Evidence Accumulation Clustering method, EEAC. Finally, we use the hierarchical single-link clustering to extract the final clusters from this matrix.

Since goodness of a cluster is determined by all the data points, the goodness function $g_{j}\left(C_{i}, D\right)$ depends on both the cluster $C_{i}$ and the entire dataset $D$, instead of $C_{i}$ alone. The stability as measure of cluster goodness is used in [9]. Cluster stability reflects the variation in the clustering results under perturbation of the data by resampling. A stable cluster is one that has a high likelihood of recurrence across multiple applications of the clustering method. Stable clusters are usually preferable, since they are robust with respect to minor changes in the dataset [10].

Now assume that we want to compute the stability of cluster $C_{i}$. In this method first a set of partitionings over resampled datasets is provided which is called the 
reference set. In this notation $D$ is resampled data and $P(D)$ is a partitioning over $D$. Now, the problem is: "How many times is the cluster $C_{i}$ repeated in the reference partitions?" Denote by $\mathrm{NMI}\left(C_{i}, P(D)\right)$, the Normalized Mutual Information between the cluster $C_{i}$ and a reference partition $P(D)$. Most previous works only compare a partition with another partition [13]. However, the stability used in [10] evaluates the similarity between a cluster and a partition by transforming the cluster $C_{i}$ to a partition and employing common partition to partition methods. To illustrate this method let $P_{1}=P^{\mathrm{a}}=\left\{C_{i}, D / C_{i}\right\}$ be a partition with two clusters, where $D / C_{i}$ denotes the set of data points in $D$ that are not in $C_{i}$. Then we may compute a second partition $P_{2}$ $=P^{\mathrm{b}}=\left\{C^{*}, D / C^{*}\right\}$, where $C^{*}$ denotes the union of all "positive" clusters in $P(D)$ and others are in $D / C^{*}$. A cluster $C_{j}$ in $P(D)$ is positive if more than half of its data points are in $C_{i}$. Now, define $\operatorname{NMI}\left(C_{i}, P(D)\right)$ by $\operatorname{NMI}\left(P^{a}, P^{b}\right)$ which is defined in [6]. This computation is done between the cluster $C_{i}$ and all partitions available in the reference set. $N M I_{i}$ stands for the stability of cluster $C_{i}$ with respect to the $i$-th partition in reference set. The total stability of cluster $C_{i}$ is defined as:

$$
\operatorname{Stability}\left(C_{i}\right)=\frac{1}{M} \sum_{i=1}^{M} N M I_{i}
$$

where $M$ is the number of partitions available in reference set. This procedure is applied for each cluster of every primary partition.

Here a drawback of computing stability is introduced and an alternative approach is suggested which is named Max method. Fig. 1 shows two primary partitions for which the stability of each cluster is evaluated. In this example K-means is applied as the base clustering algorithm with $\mathrm{K}=3$. For this example the number of all partitions in the reference set is 40 . In 36 partitions the result is relatively similar to Fig 1a, but there are four partitions in which the top left cluster is divided into two clusters, as shown in Fig 1b. Fig 1a shows a true clustering. Since the well separated cluster in the top left corner is repeated several times (90\% repetition) in partitionings of the reference set, it has to acquire a great stability value (but not equal to 1), however it acquires the stability value of 1 . Because the two clusters in right hand of Fig 1a are

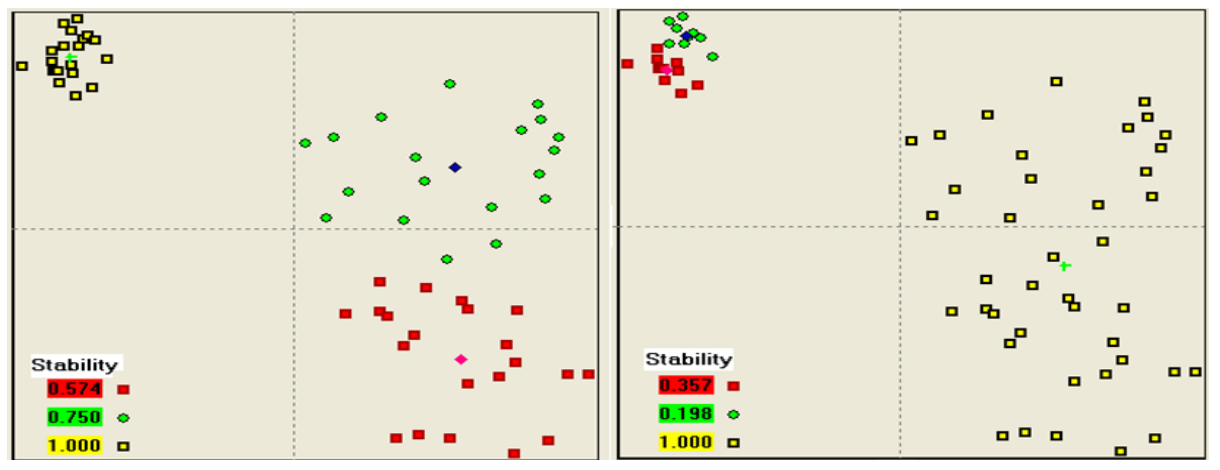

Fig. 1. Two primary partitions with $\mathrm{k}=3$. (a) True clustering. (b) Spurious clustering. 
relatively joined and sometimes they are not recognized in the reference set as well, they have less stability value. Fig. 1.b shows a spurious clustering which the two right clusters are incorrectly merged. Since a fixed number of clusters are forced in the base algorithm, the top left cluster is divided into two clusters. Here the drawback of the stability measure is apparent rarely. Although it is obvious that this partition and the corresponding large cluster on the right reference set (10\% repetition), the stability of this cluster is evaluated equal to 1 . Since the NMI is a symmetric equation, the stability of the top left cluster in Fig 1.a is exactly equal to the large right cluster in Fig 1.b; however they are repeated $90 \%$ and $10 \%$, respectively. In other words, when two clusters are complements of each other, their stabilities are always equal. This drawback is seen when the number of positive clusters in the considered partition of reference set is greater than 1 . It means when the cluster $\mathrm{C}^{*}$ is obtained by merging two or more clusters, undesirable stability effects occur.

To solve this problem we allow only one cluster in reference set to be considered as the $C^{*}$ (i.e. only the most similar cluster) and all others are considered as $D / C^{*}$. In this method the problem is solved by eliminating the merged clusters.

In the following step, the selected clusters are used to construct the co-association matrix. In the EAC method the $\mathrm{m}$ primary results from resampled data are accumulated in an $n \times n$ co-association matrix. Each entry in this matrix is computed from this equation:

$$
C(i, j)=\frac{n_{i, j}}{m_{i, j}}
$$

where $n_{i j}$ counts the number of clusters shared by objects with indices $\mathrm{i}$ and $\mathrm{j}$ in the partitions over the primary $B$ clusterings. Also $m_{i j}$ is the number of partitions where this pair of objects is simultaneously present. There are only a fraction of all primary clusters available, after thresholding. So, the common EAC method cannot truly recognize the pairwise similarity for computing the co-association matrix. In our novel method (Extended Evidence Accumulation Clustering, or EEAC) each entry of the co-association matrix is computed by:

$$
C(i, j)=\frac{n_{i, j}}{\max \left(n_{i}, n_{j}\right)}
$$

where $n_{i}$ and $n_{j}$ are the number present in remaining (after stability thresholding) clusters for the $i$-th and $j$-th data points, respectively. Also, $n_{i j}$ counts the number of remaining clusters which are shared by both data points indexed by $i$ and $j$, respectively.

\section{Experimental Results}

This section reports and discusses the empirical studies. The proposed method is examined over 5 different standard datasets. Brief information about the used datasets is available in [11]. All experiments are done over the normalized features. It means each feature is normalized with mean of 0 and variance of $1, \mathrm{~N}(0,1)$. All of them are reported over means of 10 independent runs of algorithm. The final performance of 
the clustering algorithms is evaluated by re-labeling between obtained clusters and the ground truth labels and then counting the percentage of the true classified samples. Table 1 shows the performance of the proposed method comparing with most common base and ensemble methods.

The first four columns of Table 1 are the results of some base clustering algorithms. The results show that although each of these algorithms can obtain a good result over a specific dataset, it does not perform well over other datasets. The four last columns show the performance of some ensemble methods in comparison with the proposed one. Taking a glance at the last four columns in comparison with the first four columns shows that the ensemble methods do better than the simple based algorithms in the case of performance and robustness along with different datasets. The first column of the ensemble methods is the results of an ensemble of $100 \mathrm{~K}$ means which is fused by EAC method. The $90 \%$ sampling from dataset is used for creating diversity in primary results. The sub-sampling (without replacement) is used as the sampling method. Also the random initialization of the seed points of K-means algorithm helps them to be more diverse. The single linkage algorithm is applied as consensus function for deriving the final clusters from co-association matrix. The second column from ensemble methods is the full ensemble which uses several clustering algorithms for generating the primary results. Here, $70 \mathrm{~K}$-means with the above mentioned parameters in addition to 30 linkage methods provide the primary results. The third column of Ensemble Methods is consensus partitioning using EEAC algorithm of top $33 \%$ stable clusters, employing NMI method as measure of stability. The fourth column of the ensemble methods is also consensus partitioning using EEAC algorithm of top 33\% stable clusters, employing max method as measure of stability.

Table 1. Experimental results

\begin{tabular}{||c||c|c|c|c||c|c|c|c||}
\hline \multicolumn{1}{|||}{} & \multicolumn{5}{|c||}{ Simple Methods (\%) } & \multicolumn{3}{c||}{ Ensemble Methods (\%) } \\
\hline \hline Dataset & $\begin{array}{c}\text { Single } \\
\text { Linkage }\end{array}$ & $\begin{array}{c}\text { Average } \\
\text { Linkage }\end{array}$ & $\begin{array}{c}\text { Complete } \\
\text { Linkage }\end{array}$ & Kmeans & $\begin{array}{c}\text { Kmeans } \\
\text { Ensemble }\end{array}$ & $\begin{array}{c}\text { Full } \\
\text { Ensemble }\end{array}$ & $\begin{array}{c}\text { Cluster } \\
\text { Selection by } \\
\text { NMI Method }\end{array}$ & $\begin{array}{c}\text { Cluster } \\
\text { Selection by } \\
\text { max Method }\end{array}$ \\
\hline \hline Wine & 37.64 & 38.76 & 83.71 & 96.63 & 96.63 & 97.08 & 97.75 & 97.44 \\
\hline Breast-C & 65.15 & 70.13 & 94.73 & 95.37 & 95.46 & 95.10 & 95.75 & 96.49 \\
\hline Yeast & 34.38 & 35.11 & 38.91 & 40.20 & 45.46 & 47.17 & 47.17 & $\mathbf{5 1 . 2 7}$ \\
\hline Glass & 36.45 & 37.85 & 40.65 & 45.28 & 47.01 & 47.83 & $\mathbf{4 8 . 1 3}$ & 47.35 \\
\hline Bupa & 57.68 & 57.10 & 55.94 & 54.64 & 54.49 & 55.83 & 58.09 & $\mathbf{5 8 . 4 0}$ \\
\hline
\end{tabular}

\section{Conclusion and Future Works}

In this paper a new clustering ensemble framework is proposed which is based on a subset of total primary spurious clusters. Also a new alternative method for common 
NMI is suggested. Since the quality of the primary clusters are not equal and presence of some of them can even yield to lower performance, here a method to select a subset of more effective clusters is proposed. A common cluster validity criterion which is needed to derive this subset is based on normalized mutual information. In this paper some drawbacks of this criterion is discussed and a method is suggested which is called max mehod. The experiments show that the proposed framework commonly outperforms in comparison with the full ensemble; however it uses just $33 \%$ of primary clusters. Also the proposed max criterion does slightly better than NMI criterion generally. Because of the symmetry which is concealed in NMI criterion and also in NMI based stability, it yields to lower performance whenever symmetry is also appeared in the dataset. Another innovation of this chapter is a method for constructing the co-association matrix where some of clusters and respectively some of samples do not exist in partitions. This new method is called Extended Evidence Accumulation Clustering, EEAC.

\section{References}

1. Alizadeh, H., Minaei-Bidgoli, B., Parvin, H., Mohsen, M.: An Asymmetric Criterion for Cluster Validation. In: International Conference on Industrial, Engineering and Other Applications of Applied Intelligent Systems (IEA/AIE 2011). LNCS. Springer, Heidelberg (2011)

2. Ayad, H., Kamel, M.S.: Cumulative Voting Consensus Method for Partitions with a Variable Number of Clusters. IEEE Trans. on Pattern Analysis and Machine Intelligence 30(1), 160-173 (2008)

3. Faceli, K., Marcilio, C.P., Souto, D.: Multi-objective Clustering Ensemble. In: Proceedings of the Sixth International Conference on Hybrid Intelligent Systems, HIS 2006 (2006)

4. Fern, X.Z., Lin, W.: Cluster Ensemble Selection. In: Jonker, W., Petković, M. (eds.) SDM 2008. LNCS, vol. 5159. Springer, Heidelberg (2008)

5. Fred, A., Jain, A.K.: Data Clustering Using Evidence Accumulation. In: Proc. of the 16th Intl. Conf. on Pattern Recognition, ICPR 2002, Quebec City, pp. 276-280 (2002)

6. Fred, A., Jain, A.K.: Combining Multiple Clusterings Using Evidence Accumulation. IEEE Trans. on Pattern Analysis and Machine Intelligence 27(6), 835-850 (2005)

7. Fred, A., Jain, A.K.: Learning Pairwise Similarity for Data Clustering. In: Proc. of the 18th Int. Conf. on Pattern Recognition, ICPR 2006 (2006)

8. Fred, A., Lourenco, A.: Cluster Ensemble Methods: from Single Clusterings to Combined Solutions. Studies in Computational Intelligence (SCI) 126, 3-30 (2008)

9. Lange, T., Braun, M.L., Roth, V., Buhmann, J.M.: Stability-based model selection. In: Advances in Neural Information Processing Systems, vol. 15. MIT Press, Cambridge (2003)

10. Law, M.H.C., Topchy, A.P., Jain, A.K.: Multiobjective data clustering. In: Proc. of IEEE Conference on Computer Vision and Pattern Recognition, vol. 2, pp. 424-430 (2004)

11. Newman, C.B.D.J., Hettich, S., Merz, C.: UCI repository of machine learning databases (1998), http: / / www. ics.uci.edu/ mlearn/MLSummary.html

12. Roth, V., Lange, T., Braun, M., Buhmann, J.: A Resampling Approach to Cluster Validation. In: Intl. Conf. on Computational Statistics, COMPSTAT (2002)

13. Strehl, A., Ghosh, J.: Cluster ensembles - a knowledge reuse framework for combining multiple partitions. Journal of Machine Learning Research 3, 583-617 (2002) 